\title{
The negative magnetic pressure effect in stratified turbulence
}

\author{
K. Kemel ${ }^{1,2}$, A. Brandenburg ${ }^{1,2}$, N. Kleeorin ${ }^{3}$, and I. Rogachevskii ${ }^{3}$ \\ ${ }^{1}$ NORDITA, AlbaNova University Center, Roslagstullsbacken 23, SE-10691 Stockholm, Sweden \\ ${ }^{2}$ Department of Astronomy, Stockholm University, SE-10691 Stockholm, Sweden \\ ${ }^{3}$ Department of Mechanical Engineering, Ben-Gurion University of the Negev, \\ POB 653, Beer-Sheva 84105, Israel
}

\begin{abstract}
While the rising flux tube paradigm is an elegant theory, its basic assumptions, thin flux tubes at the bottom of the convection zone with field strengths two orders of magnitude above equipartition, remain numerically unverified at best. As such, in recent years the idea of a formation of sunspots near the top of the convection zone has generated some interest. The presence of turbulence can strongly enhance diffusive transport mechanisms, leading to an effective transport coefficient formalism in the mean-field formulation. The question is what happens to these coefficients when the turbulence becomes anisotropic due to a strong large-scale mean magnetic field. It has been noted in the past that this anisotropy can also lead to highly non-diffusive behavior. In the present work we investigate the formation of large-scale magnetic structures as a result of a negative contribution of turbulence to the large-scale effective magnetic pressure in the presence of stratification. In direct numerical simulations of forced turbulence in a stratified box, we verify the existence of this effect. This phenomenon can cause formation of large-scale magnetic structures even from initially uniform large-scale magnetic field.
\end{abstract}

Keywords. Turbulence, MHD, sunspots

\section{Introduction}

The standard explanation for the appearance of strong magnetic fields at the solar surface involves the coherent rise of a tachocline-generated magnetic flux tube through the solar convection zone. Flux tube emergence simulations do give very promising results (Rempel et al. 2009), the paradigm looks elegant and is very textbook friendly, but some of its assumptions are problematic: the integrity of flux tubes, their rise, and even their very existence.

So far, numerical simulations have failed to produce the assumed thin magnetic flux tubes in the tachocline (Cattaneo et al. 2006; Parker 2009). Magnetic buoyancy as the driving transport mechanism through the convection zone can be dominated by downward pumping (Nordlund et al. 1992; Tobias et al. 1998). For the tubes to remain intact, strongly super-equipartition field strengths and strong twists are required (Fan 2001).

Thus, it seems not unreasonable to explore alternative mechanisms of formation of strong magnetic fields at the solar surface. A number of positive arguments has been put forward (Brandenburg 2005) in favour of formation of sunspots from local flux concentrations near the surface. In mean-field models, such magnetic instabilities have been produced by introducing a magnetic dependence of the thermal eddy diffusivity (Kitchatinov \& Mazur 2000) and the viscous stress tensor (Brandenburg et al. 2010a).

Turbulence generally is associated with enhanced transport effects. However, it also exhibits non-diffusive behaviour, generating magnetic field on much larger scales than the driving scale. An example is turbulent dynamos that are able to produce large-scale 
magnetic fields (Brandenburg \& Subramanian 2005). In a stratified layer, magnetic fields tend to become buoyantly unstable: assuming a constant temperature across the magnetic flux tube, pressure balance implies lower densities in regions of stronger magnetic field. Now one can wonder what the role of turbulent pressure is in such an equilibrium if the magnetic structures extend over several turbulent eddies. The turbulent pressure associated with the convective fluid motions is certainly not negligible and is strongly affected by the background magnetic field. The latter can be seen by evaluating the total turbulent dynamic pressure, here given for the isotropic case:

$$
P_{\text {turb }}=\frac{1}{3} \overline{\rho u^{2}}+\frac{1}{6} \overline{b^{2}} / \mu_{0},
$$

here $\boldsymbol{u}$ and $\boldsymbol{b}$ are the velocity and magnetic fluctuations, respectively, $\mu_{0}$ the vacuum permeability and $\rho$ the fluid density. Overbars indicate ensemble averaging. As shown in direct numerical simulations (Brandenburg et al. 2010a, hereafter referred to as BKR), in forced turbulence with imposed uniform large-scale magnetic field, the total turbulent energy is approximately conserved in this parameter regime.

$$
\frac{1}{2} \overline{\rho u^{2}}+\frac{1}{2} \overline{b^{2}} / \mu_{0} \equiv E_{\text {tot }} \approx \text { const. }
$$

As a result, one finds a reversed feedback from the magnetic fluctuations on the turbulent pressure:

$$
P_{\text {turb }}=-\frac{1}{6} \overline{b^{2}} / \mu_{0}+2 E_{\text {tot }} / 3
$$

(Kleeorin et al. 1990; Rogachevskii \& Kleeorin 2007, hereafter referred to as RK07). One can see that the effective mean magnetic pressure force is reduced and can, in a certain parameter range, be reversed. This effect would then counteract the aforementioned buoyancy instability in the presence of turbulence. RK07 have suggested that the reversed feedback instability could lead to the formation of magnetic flux concentrations near the solar surface.

Mean-field magnetohydromagnetic simulations by BKR confirmed the basic phenomenon of magnetic flux concentration by the effect of turbulence on the mean Lorentz force and for sufficient stratification, a linear instability was found. Direct numerical simulations (DNS) by BKR confirmed the reversed feedback phenomenology, but did not address the effect of stratification. This is one of the important additions of the more recent work of Brandenburg et al. (2010b), of which we report here the main highlights.

\section{DNS model and analysis}

DNS of forced turbulence were performed in a cubic computational domain of size $L^{3}$. For an isothermal equation of state and a constant vertical gravitational acceleration $g$, one finds an exponentially stratified density:

$$
\rho=\rho_{0} \exp \left(-z / H_{\rho}\right),
$$

where $H_{\rho}=c_{s}^{2} / g$ is the constant density scale height, $c_{s}$ is the isothermal sound speed and $\rho_{0}$ a normalisation factor. We choose $k_{1} H_{\rho}=1$, where $k_{1}=2 \pi / L$, the smallest wavenumber. The density contrast then corresponds to $\exp 2 \pi \approx 535$.

We solve the equations of compressible magneto-hydrodynamics in the form

$$
\begin{gathered}
\rho \frac{D \boldsymbol{U}}{D t}=\boldsymbol{J} \times \boldsymbol{B}-c_{s}^{2} \nabla \ln \rho+\nabla \cdot(2 \nu \rho \mathbf{S})+\rho(\boldsymbol{f}+\boldsymbol{g}), \\
\frac{\partial \boldsymbol{A}}{\partial t}=\boldsymbol{U} \times \boldsymbol{B}+\eta \nabla^{2} \boldsymbol{A},
\end{gathered}
$$




$$
\frac{\partial \rho}{\partial t}=-\nabla \cdot \rho \boldsymbol{U}
$$

where $\nu$ and $\eta$ are kinematic viscosity and magnetic diffusivity, respectively. Furthermore, $\boldsymbol{B}=\boldsymbol{B}_{0}+\nabla \times \boldsymbol{A}$ is the magnetic field consisting of a uniform mean field, $\boldsymbol{B}_{0}=\left(0, B_{0}, 0\right)$, and a nonuniform part that is represented in terms of the magnetic vector potential $\boldsymbol{A}$, $\boldsymbol{J}=\nabla \times \boldsymbol{B} / \mu_{0}$ is the current density, and $\boldsymbol{S}_{i j}=\frac{1}{2}\left(U_{i, j}+U_{j, i}\right)-\frac{1}{3} \delta_{i j} \nabla \cdot \boldsymbol{U}$ is the traceless rate of strain tensor, where commas denote partial differentiation. The turbulence is driven with a forcing function $\boldsymbol{f}$ that consists of random plane non-polarized waves with an average wavenumber $k_{\mathrm{f}}=5 k_{1}$. The forcing strength is arranged such that the turbulent rms velocity, $u_{\mathrm{rms}}=\left\langle\boldsymbol{u}^{2}\right\rangle^{1 / 2}$, is around $0.1 c_{s}$. This value is small enough so that compressibility effects are weak.

In order to characterize our simulations, a set of dimensionless parameters is defined. The Reynolds number is given by $\operatorname{Re}=u_{\mathrm{rms}} / \nu k_{\mathrm{f}}$ and is of the order 120 in the simulations. The magnetic Prandtl number is $\pm=\nu / \eta$, although in reality this parameter is much smaller than unity; we choose here $\pm=0.5-8$ in order to achieve higher values for the magnetic Reynolds number. The equipartition field strength $B_{\text {eq }}$ is defined as a function of $z$ and the imposed fields are normalised against $B_{\text {eq } 0}$, the equipartition strength in the middle of the domain:

$$
B_{\text {eq }}(z)=\left(\mu_{0} \overline{\rho \boldsymbol{u}^{2}}\right)^{1 / 2}, \quad B_{\text {eq } 0}=\left(\mu_{0} \rho_{0}\right)^{1 / 2} u_{\mathrm{rms}} .
$$

The boundary conditions are stress-free perfect conductors at the top and bottom of the domain, periodicity in the horizontal direction. The simulations are performed with the PENCIL CoDE†, which uses sixth-order explicit finite differences in space and a third-order accurate time stepping method (Brandenburg \& Dobler 2002).

The contribution to the mean momentum density by the fluctuations is

$$
\bar{\Pi}_{i j}^{f}=\bar{\rho} \overline{u_{i} u_{j}}+\frac{1}{2} \delta_{i j} \overline{b^{2}}-\overline{b_{i} b_{j}},
$$

where the overbars now indicate horizontal averages. The influence of the mean magnetic field can be found by subtracting the contributions that are present in the absence of a uniform background magnetic field and can be modelled by the following ansatz (RK07)

$$
\bar{\Pi}_{i j}^{f, \bar{B}}-\bar{\Pi}_{i j}^{f, \overline{0}}=-\left(\frac{1}{2} \delta_{i j} q_{p}+e_{i} e_{j} q_{e}\right) \overline{\boldsymbol{B}}^{2}+q_{s} \bar{B}_{i} \bar{B}_{j},
$$

which will then appear in the effective mean Lorentz force

$$
\rho \boldsymbol{F}_{i}^{M}=-\nabla_{j}\left(\delta_{i j} \overline{\boldsymbol{B}}^{2}+\bar{B}_{i} \bar{B}_{j}+\bar{\Pi}_{i j}^{f, \bar{B}}-\bar{\Pi}_{i j}^{f, \overline{0}}\right) .
$$

Thus the total effective magnetic pressure of the mean field is given by $1-q_{p}(\bar{B}) \overline{\boldsymbol{B}}^{2}$ As $\overline{\boldsymbol{B}} \approx(0, \bar{B}, 0)$, and assuming no small-scale dynamo, we can determine $q_{p}$ from

$$
\rho\left(\overline{u_{x}^{2}}-\overline{u_{0 x}^{2}}\right)+\frac{1}{2} \overline{\boldsymbol{b}^{2}}-\overline{b_{x}^{2}}=-\frac{1}{2} q_{p} \overline{\boldsymbol{B}}^{2} .
$$

\section{Results}

We compare the effective magnetic pressure of the mean field with the turbulent kinetic energy density (Fig. 1a) and see that the resulting contribution of stratified turbulence

$$
\dagger \text { http://pencil-code.googlecode.com }
$$



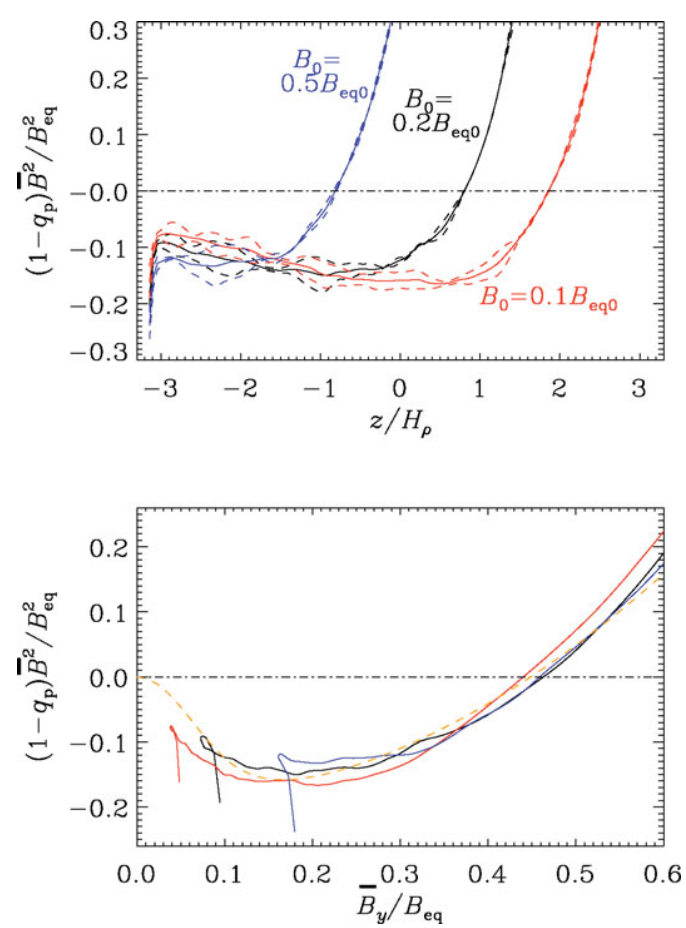

Figure 1. Normalized effective mean magnetic pressure for $B_{0}=0.1 B_{\text {eq } 0}, B_{0}=0.2 B_{\text {eq } 0}$, and $B_{0}=0.5 B_{\text {eq } 0}$ using $R e=120$. Left: as a function of depth Right: as function of the local value of the ratio of $B_{0} / B_{\mathrm{eq}}(z)$. Note that the curves for the different imposed field strengths collapse onto a single dependence and agree very well with the fit by BKR (dashed line). Adapted from Brandenburg et al. (2010b).

to the effective magnetic pressure is negative in a large part of the domain. Plotting the ratio of effective magnetic pressure to kinetic energy density as a function of the imposed uniform horizontal magnetic field divided by the equipartition field strength (Fig. 1b), collapses the observations from simulations with different imposed field strengths into a single dependence of $q_{p}$ on $\overline{B_{y}} / B_{\text {eq }}$. This result agrees with analytical calculations by RK07 and a fit based on simulations by BKR. The effect is fairly robust under an increase of the magnetic Prandtl number (Fig. 2a). While the reduced effective magnetic pressure is observed, the formation of local magnetic field concentrations as observed in mean-field simulations, has not yet been found in DNS (Fig. 2b).

\section{Discussion}

The DNS have shown that for an isothermal atmosphere with strong density stratification, the turbulent pressure is decreased due to a negative feedback from magnetic fluctuation generation, resulting in a negative effective mean magnetic pressure. The dependence on the ratio of imposed field to local equipartition field agrees with results obtained from analytic theory (RK07) and direct numerical simulations (BKR). The results are robust when changing the strength of the imposed field and the magnetic Prandtl number.

However, the simulations do not show any obvious signs of a large-scale instability that should result in magnetic flux concentrations, as was expected from mean-field calculations. A possible explanation for this discrepancy could be the simplicity of the ansatz 

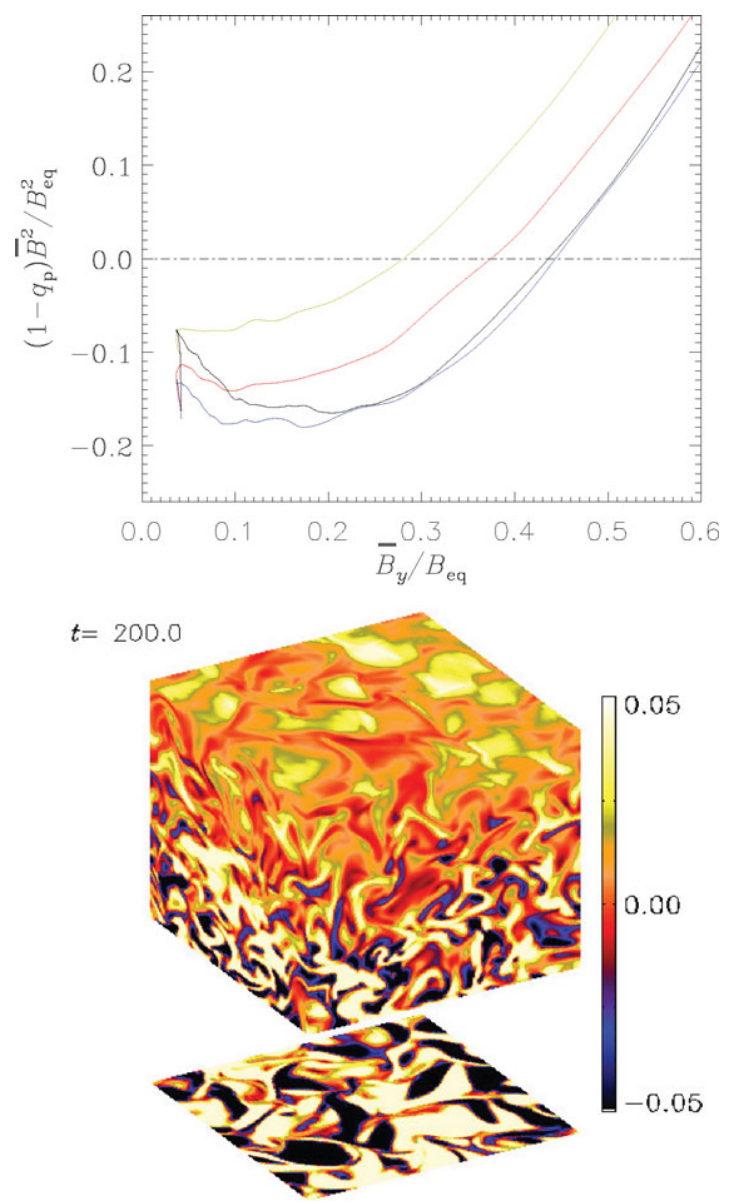

Figure 2. Left: Normalized effective mean magnetic pressure as a function of $B_{0} / B_{\text {eq }}(z)$ for $B_{0}=0.1 B_{\text {eq0 }}$, and varying magnetic Prandtl number, $\pm=0.05$ (black), 2 (blue), 4 (red) and 8 (yellow). Right: Visualisation of $B_{y}-B_{0}$ on the periphery of the computational domain $B_{0}=0.1 B_{\text {eq } 0}$, and $\pm=2$.

for the effective Lorentz force. Indeed, early simulations of Tao et al. (1998) produced clear signs of flux separation into magnetised and unmagnetised regions in convection simulations at large aspect ratio. An increase of scale separation might alleviate the effect of higher order terms. Future work should incorporate this increase as well as a wider scan of the parameter regime and ultimately the inclusion of radiative transfer, as was done in simulations of Kitiashvili et al. (2010), which showed flux concentrations in the presence of a vertical field.

\section{Acknowledgements}

We acknowledge the allocation of computing resources provided by the Swedish National Allocations Committee at the Center for Parallel Computers at the Royal Institute of Technology in Stockholm and the National Supercomputer Centers in Linköping as well as the Norwegian National Allocations Committee at the Bergen Center for Computational Science. This work was supported in part by the European Research Council under the AstroDyn Research Project No. 227952 and the Swedish Research Council 
Grant No. 621-2007-4064. NK and IR thank NORDITA for hospitality and support during their visits.

\section{References}

Brandenburg, A., 2005, Astrophys. J., 625, 539

Brandenburg, A. \& Dobler, W., 2002, Comp. Phys. Comm.147, 471

Brandenburg, A. \& Subramanian, K., 2005, Phys. Rep., 417, 1

Brandenburg, A., Kleeorin, N., \& Rogachevskii, I., 2010, Astron. Nachr., 331, 5 (BKR)

Brandenburg, A., Kemel, K., Kleeorin, N., \& Rogachevskii, I., 2010, arXiv:1005.5700

Cattaneo, F., Brummell, N. H., \& Cline, K. S., 2006, Mon. Not. Roy. Astron. Soc., 365, 727

Fan, Y., 2001, Astrophys. J., 546, 509

Kitchatinov, L. L. \& Mazur, M. V., 2000, Solar Phys., 191, 325

Kitiashvili, I. N., Kosovichev, A. G., Wray, A. A., \& Mansour, N. N., 2010, Astrophys. J., 719, 307

Kleeorin, N. I., Rogachevskii, I. V., \& Ruzmaikin, A. A., 1990, Sov. Phys., 70, 878

Nordlund, Å., Brandenburg, A., Jennings, R. L., Rieutord, M., Ruokolainen, J., Stein, R. F., \& Tuominen, I., 1992, Astrophys. J., 392, 647

Parker, E. N., 2009, Space Sci. Rev., 144, 15

Rempel, M., Schüssler, M., \& Knölker, M., 2009, Astrophys. J., 691, 640

Rogachevskii, I. \& Kleeorin, N., 2007, Phys. Rev. E, 76, 056307(RK07)

Tao, L., Weiss, N. O., Brownjohn, D. P., \& Proctor, M. R. E., 1998, Astrophys. J., 496, L39

Tobias, S. M., Brummell, N. H., Clune, T. L., \& Toomre, J., 1998, Astrophys. J., 502, L177 